Document downloaded from:

http://hdl.handle.net/10251/145555

This paper must be cited as:

Gonzalez-German, IT.; Sala, A.; Bernal Reza, MÁ. (01-0). A generalised integral polynomial Lyapunov function for nonlinear systems. Fuzzy Sets and Systems. 356:77-91. https://doi.org/10.1016/j.fss.2018.02.005

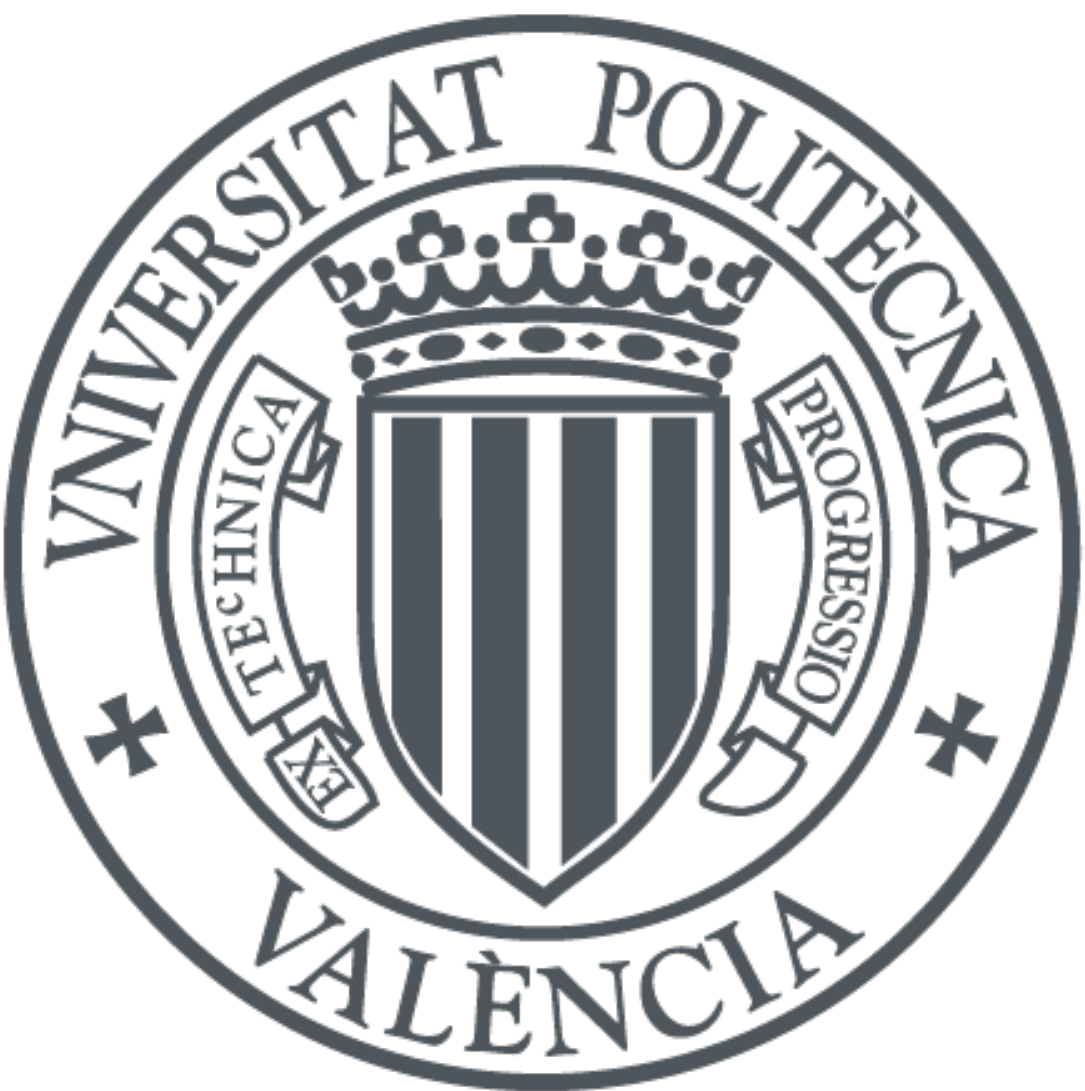

The final publication is available at

https://doi.org/10.1016/j.fss.2018.02.005

Copyright Elsevier

Additional Information 


\title{
A Generalised Integral Polynomial Lyapunov Function for Nonlinear Systems
}

\author{
Temoatzin González ${ }^{\mathrm{b}, *}$, Antonio Sala ${ }^{\mathrm{b}}$, Miguel Bernal ${ }^{\mathrm{a}}$ \\ ${ }^{a}$ Departamento de Ingeniería Eléctrica y Electrónica, Instituto Tecnológico de Sonora, 5 \\ de Febrero 818 Sur, 85000, Ciudad Obregón, Mexico \\ ${ }^{b}$ Instituto Universitario de Automática e Informática Industrial, Universidad Politécnica \\ de Valencia, Camino de Vera S/N, 46022, Valencia, Spain
}

\begin{abstract}
This work generalises the line-integral Lyapunov function in (Rhee \& Won, 2006) for stability analysis of continuous-time nonlinear models expressed as fuzzy systems. The referred result applied only to Takagi-Sugeno representations, and required memberships to be a tensor-product of functions of a single state; these are generalised here so that membership arguments can be arbitrary polynomials of the state variables; in this way, systems for which earlier results cannot be applied are now covered. Both the modelling and the integral terms appearing in the Lyapunov functions are generalised to a fuzzy polynomial case. Illustrative examples show the advantage of the proposed method against previous literature, even in the TS case.
\end{abstract}

Keywords: Line-Integral Lyapunov Function, Fuzzy Polynomial Models, Sum of Squares, Stability Analysis, Nonlinear Systems

\section{Introduction}

Stability analysis of nonlinear systems has benefited in the last twenty years from a representation as a combination of linear models, denoted as Takagi-Sugeno (TS) [1] or quasi-LPV [2] representations. Obtaining such models via the sector nonlinearity approach [3] allows to exactly rewrite a nonlinear system as a convex sum of linear models within a compact set

\footnotetext{
${ }^{*}$ Corresponding author

Email addresses: temoatzin.g@gmail.com (Temoatzin González), asala@isa.upv.es (Antonio Sala), miguel.bernal@itson.edu.mx (Miguel Bernal)
} 
of the state space (modeling region), the nonlinearities being captured in so-called membership functions (MFs) which are in general state-dependent and hold the convex sum property [4]. Later on, in [5, 6], via the Taylorseries approach, the sector nonlinearity idea was extended to polynomial fuzzy models: this representation expresses non-polynomial nonlinearities as an equivalent convex sum of polynomial consequents, blended together by MFs.

When a TS model is available, stability analysis and controller design are usually performed via the direct Lyapunov method, which usually leads to conditions in the form of linear matrix inequalities (LMIs) [4]. LMI conditions are highly appreciated as their feasibility can be decided via convex optimization techniques [7]. Different classes of Lyapunov functions have been used to overcome the conservatism of the common quadratic one, first proposed in [8]: piecewise [9, 10], fuzzy (also unspecifically known as "nonquadratic") [11, 12], and fuzzy line-integral (LI) [13]. Other recent proposals, intentionally left out of this quest, are based on polyhedron manipulations and set-invariance considerations [14; these proposals avoid the need of fixing a structure of a Lyapunov function and, importantly, are asymptotically exact (under some conditions) for the TS case; however, they cannot be extended to the fuzzy-polynomial setup below.

In [15, 16, the quadratic LMI/TS framework was extended to the sum-ofsquares (SOS) approach [17, 18, which use polynomial Lyapunov functions for stability analysis of nonlinear systems in fuzzy-polynomial form, posing SOS conditions which are actually reducible to LMIs. Later on, a fuzzy polynomial Lyapunov function was employed to generalize results for fuzzy polynomial models [19]. In that work, the time-derivative of the MFs is a priori bounded by polynomials of the state, thus obtaining a fuzzy polynomial model of the time derivative of the MFs. As a last option on these issues, [20] presented a piecewise Lyapunov function defined by the minimum or maximum of polynomials.

The widely-cited work [13 proposed an interesting fuzzy line-integral Lyapunov function, presenting LMI stability conditions which are global and avoided the time derivative of the MFs. The goal of this paper is generalising the fuzzy LI approach in the above-referred work to the polynomial case, incorporating, too, the additional refinements outlined in our preliminary conference paper [21]: it turns out that path independency conditions for line integrals are automatically verified if the integral can be expressed as a sum of single-variable terms. Let us, next, discuss in detail the motivation 
behind our proposal.

In [13], a Lyapunov function with integral terms was pursued. However, since such Lyapunov function depended on necessary path-independence conditions, the approach was only applicable to a specific class of TS models where the MFs are a tensor-product expression [22] of at most $n$ nonlinear components where each of them depends exactly on one state variable. For this class of models, only the "diagonal" terms of the Lyapunov function were actually using fuzzy summations and, moreover, if the MFs depend on multiple variables and cannot be factorised, e.g., $w_{i}\left(x_{1}+x_{2}\right) \neq \alpha\left(x_{1}\right) \beta\left(x_{2}\right)$, the approach in [13] cannot be directly applied.

In order to generalize the class of TS model on where the LI approach can be applied, the LF in [13] is expressed as a sum of single-variable integrals, as above mentioned. Resorting to such parametrisation, path-independence conditions are automatically fulfilled. This was the idea behind a preliminary result presented in [21], introducing a larger class of path-independent lineintegral Lyapunov functions whenever the MFs depended on an arbitrary set of linear functions of the system states. Other refinements on the work of [13] can be found in [23, 24]; they exploit a relaxation from a determinant formula which applies only to second-order TS systems, but do not correspond to the point of view hereby adopted (pursuing results applicable to higher-order systems).

Motivated by the ideas above, this paper presents a Polynomial Lyapunov function including integral terms, for the stability analysis of a class of nonlinear models so the results in [13, 21] are a particular case. The results in this manuscript apply to nonlinear systems that can be expressed in terms of single-variable non-polynomial nonlinearities with a polynomial argument.

The paper is organized as follows: section 2 presents the classical sector nonlinearity approach to obtain TS models, previous results about the line-integral Lyapunov approach, and a review on the standard polynomial fuzzy framework; section 3 develops the main result, where a new Polynomial+Integral Lyapunov function is built; section 4 gives some examples to illustrate the effectiveness of the proposed approach; finally, discussion, concluding remarks and ideas for future work are given in sections 5 and 6 . 


\section{Preliminaries and problem statement}

\subsection{Takagi-Sugeno models}

Consider a nonlinear system:

$$
\dot{x}(t)=h(x(t))
$$

with $x \in \mathbb{R}^{n}$ being the state vector, and $x=0$ being an equilibrium point, i.e., $h(0)=0$. Let us assume that $h(\cdot)$ can be expressed in the form:

$$
\dot{x}(t)=\tilde{h}(\eta(x), x),
$$

where $\tilde{h}(\cdot)$ is linear in $x(t)$ and multiaffine in $\eta(x) \in \mathbb{R}^{q}$, where

$$
\eta(x)=\left[\eta_{1}(x) \eta_{2}(x) \cdots \eta_{q}(x)\right]^{T}
$$

is a set of continuous functions which collects all nonlinearities present in $h(\cdot)$ in (1). Then, the above model can be written as [25]:

$$
\dot{x}(t)=\tilde{f}(\eta(x)) x(t),
$$

with $\tilde{f}(\cdot): \mathbb{R}^{q} \mapsto \mathbb{R}^{n}$ being a multiaffine function in its arguments.

A well-established procedure for convex rewriting of such nonlinear systems within a compact set $\Omega \supset\{0\}$ of the state space, called the sector nonlinearity methodology [3], is available. Let us outline the main ideas of it in order to introduce notation which will be used in later developments in the paper.

Since, by continuity and compactness, the components of vector $\eta(x)$ are bounded in $\Omega$, assume $\eta_{j}(x) \in\left[\underline{\eta}_{j}, \bar{\eta}_{j}\right], j \in\{1,2, \ldots, q\}$ in $\Omega$. By defining the following weighting functions (WFs):

$$
w_{0}^{j}(x):=\frac{\bar{\eta}_{j}-\eta_{j}(x)}{\bar{\eta}_{j}-\underline{\eta}_{j}}, w_{1}^{j}(x)=1-w_{0}^{j}(x), j \in\{1,2, \ldots, q\},
$$

each nonlinearity is written as $\eta_{j}(x)=w_{0}^{j}(x) \underline{\eta}_{j}+w_{1}^{j}(x) \bar{\eta}_{j}$, with $0 \leq w_{i}^{j} \leq 1$, $w_{0}^{j}+w_{1}^{j}=1$. On the sequel, dependence of $w_{i}^{j}$ on the state $x$ will be omitted for notational brevity if clear from the context.

As $\tilde{f}$ is multiaffine, straightforward manipulations lead to a TS model with $q$ nested convex sums:

$$
\dot{x}(t)=A_{w} x(t),
$$




$$
\begin{gathered}
A_{w}:=\sum_{i_{1}=0}^{1} \sum_{i_{2}=0}^{1} \cdots \sum_{i_{q}=0}^{1} w_{i_{1}}^{1} w_{i_{2}}^{2} \cdots w_{i_{q}}^{q} A_{\left(i_{1}, i_{2}, \ldots, i_{q}\right)}, \\
A_{\left(i_{1}, i_{2}, \ldots, i_{q}\right)}=\left.\tilde{f}(\eta(x))\right|_{w_{i_{1}}^{1}=w_{i_{2}}^{2}=\cdots=w_{i_{q}}^{q}=1},
\end{gathered}
$$

with $A_{\left(i_{1}, i_{2}, \ldots, i_{q}\right)} \in \mathbb{R}^{n \times n}, i_{j} \in\{0,1\}, j \in\{1,2, \ldots, q\}$. This sort of notation for TS models corresponds to the tensor-product modelling approach [22, 26]. The reader is referred to these works for further details on the above fuzzy modelling steps, which routinely appear in systems with several nonlinearities.

Example 1. Consider the following nonlinear system:

$$
\dot{x}=\left[\begin{array}{cc}
-a-b\left(1+\cos \rho_{2}\right)+0.2 \cos \rho_{3} & -3+\cos \rho_{3}-5 \sin \rho_{1} \\
-0.5 a+0.2 b\left(1+\cos \rho_{3}\right) & -4.6+\cos \rho_{3}+\sin \rho_{1}
\end{array}\right]\left[\begin{array}{l}
x_{1} \\
x_{2}
\end{array}\right]
$$

where $\eta_{1}(x):=\sin \left(\rho_{1}(x)\right)$ with $\rho_{1}(x):=x_{2}, \eta_{2}(x):=\cos \left(\rho_{2}(x)\right)$ with $\rho_{2}(x):=$ $2 x_{2}^{2}-x_{1} x_{2}$, and $\eta_{3}(x):=\cos \left(\rho_{3}(x)\right)$ with $\rho_{3}(x):=x_{2}-4 x_{1}^{2}$. If we independently model $\sin \left(\rho_{1}\right), \cos \left(\rho_{2}\right)$, and $\cos \left(\rho_{3}\right)$ in the previous system via standard sector nonlinearity, we get the following tensor-product TS model with $2^{3}$ vertices:

$$
\dot{x}=\sum_{i_{1}=0}^{1} \sum_{i_{2}=0}^{1} \sum_{i_{3}=0}^{1} w_{i_{1}}^{1}\left(\rho_{1}\right) w_{i_{2}}^{2}\left(\rho_{2}\right) w_{i_{3}}^{3}\left(\rho_{3}\right) A_{\left(i_{1}, i_{2}, i_{3}\right)} x
$$

with

$$
\begin{gathered}
A_{000}=\left[\begin{array}{cc}
-a-0.2 & 1 \\
-0.5 a & -6.6
\end{array}\right], A_{001}=\left[\begin{array}{cc}
0.2-a & 3 \\
0.4 b-0.5 a & -4.6
\end{array}\right], A_{010}=\left[\begin{array}{cc}
-a-2 b-0.2 & 1 \\
-0.5 a & -6.6
\end{array}\right], \\
A_{011}=\left[\begin{array}{cc}
0.2-2 b-a & 3 \\
0.4 b-0.5 a & -4.6
\end{array}\right], A_{100}=\left[\begin{array}{cc}
-a-0.2 & -9 \\
-0.5 a & -4.6
\end{array}\right], A_{101}=\left[\begin{array}{cc}
0.2 . a & -7 \\
0.4 b-0.5 a & -2.6
\end{array}\right], \\
A_{110}=\left[\begin{array}{cc}
-a-2 b-0.2 & -9 \\
-0.5 a & -4.6
\end{array}\right], \quad A_{111}=\left[\begin{array}{cc}
0.2-2 b-a & -7 \\
0.4 b-0.5 a & -2.6
\end{array}\right],
\end{gathered}
$$

and WFs $w_{0}^{1}\left(\rho_{1}\right)=0.5\left(1-\sin \left(\rho_{1}\right)\right), w_{1}^{1}\left(\rho_{1}\right)=1-w_{0}^{1}\left(\rho_{1}\right), w_{0}^{2}\left(\rho_{2}\right)=0.5(1-$ $\left.\cos \left(\rho_{2}\right)\right), w_{1}^{2}\left(\rho_{2}\right)=1-w_{0}^{2}\left(\rho_{2}\right), w_{0}^{3}\left(\rho_{3}\right)=0.5\left(1-\cos \left(\rho_{3}\right)\right), w_{1}^{3}\left(\rho_{3}\right)=1-w_{0}^{3}\left(\rho_{3}\right)$.

In order to get a more compact notation, the multi-index shorthand notation from, for instance, [27, 28, will be used with $\mathbf{a}:=\left(a_{1}, a_{2}, \ldots, a_{q}\right), a_{j} \in$ $\{0 \cup \mathbb{N}\}$, and $\mathbf{b}:=\left(b_{1}, b_{2}, \ldots, b_{q}\right), b_{j} \in\{0 \cup \mathbb{N}\}, q$-dimensional multi-indices (q-tuples):

$$
\mathbf{w}_{0}^{\mathbf{a}}:=\left(w_{0}^{1}\right)^{a_{1}}\left(w_{0}^{2}\right)^{a_{2}} \cdots\left(w_{0}^{q}\right)^{a_{q}}, \quad \mathbf{w}_{1}^{\mathbf{b}}:=\left(w_{1}^{1}\right)^{b_{1}}\left(w_{1}^{2}\right)^{b_{2}} \cdots\left(w_{1}^{q}\right)^{b_{q}} .
$$


Then, the nested convex sum $A_{w}$ in (6) can be equivalently rewritten as:

$$
A_{w}=\sum_{\mathbf{j}+\mathbf{i}=\mathbf{1}} \mathbf{w}_{0}^{\mathbf{j}} \mathbf{w}_{1}^{\mathbf{i}} A_{\mathbf{i}},
$$

with $\mathbf{j}+\mathbf{i}:=\left(j_{1}+i_{1}, j_{2}+i_{2}, \ldots, j_{q}+i_{q},\right)$ being the element-wise sum of $q$ tuples, and $\mathbf{1}:=(1,1, \ldots, 1)$. For instance, in the above example $q=3$.

Well-known conditions for quadratic stability, with Lyapunov function $V(x)=x^{T} P x$, of the above model are [4:

$$
P>0, \quad P A_{\mathbf{i}}+A_{\mathbf{i}}^{T} P \leq 0 .
$$

However, these conditions are known to be conservative. Other options, called non-quadratic LF, have appeared in literature (see, for instance, 29, 30, 11]), in which the LF is in the form:

$$
V(x)=x^{T} P_{w} x=x^{T}\left(\sum_{\mathbf{j}+\mathbf{i}=\mathbf{1}} \mathbf{w}_{0}^{\mathbf{j}} \mathbf{w}_{1}^{\mathbf{i}} P_{\mathbf{i}}\right) x .
$$

However, as $\dot{V}(x)$ depends on $\dot{P}_{w}$, time-derivative bounds on the WFs are needed [30, 31] or, via chain-rule argumentations, bounds on the partial derivatives of them [32, 33]. In some cases, a cancellation-based controller design approach can be crafted to avoid the WF derivative bounds [28] in the resulting closed-loop expressions.

\subsection{Line-integral fuzzy Lyapunov Functions in prior literature}

Consider the particular case of a model (2) with $\eta_{j}(x)$ depending only on $x_{j}(t)$ and $q \leq n$. Then, from (4), each $w_{k}^{j}(\cdot)$ only depends on $x_{j}$. On the sequel, given $f: \mathbb{R}^{n} \mapsto \mathbb{R}^{h}$ notation $\nabla f$ denotes the Jacobian matrix of size $h \times n$.

In the work [13], based on line-integral considerations, the following lineintegral fuzzy Lyapunov function was proposed:

$$
V(x)=\int_{\Gamma(0, x)} f(\psi) d \psi
$$

where $\Gamma(0, x)$ was any one-dimensional path betwen 0 and $x, \psi \in \mathbb{R}^{n}$ is a dummy vector for the integral argument, and $d \psi \in \mathbb{R}^{n}$ is an infinitesimal 
displacement vector along the path, and $f(\psi)$ was given by:

$$
f(\psi)=\psi^{T} P+\left[\begin{array}{c}
\sum_{j_{1}+i_{1}=1}\left(w_{0}^{1}\right)^{j_{1}}\left(w_{1}^{1}\right)^{i_{1}} s_{i_{1}}^{1} \psi_{1} \\
\sum_{j_{2}+i_{2}=1}^{2}\left(w_{0}^{2}\right)^{j_{2}}\left(w_{1}^{2}\right)^{i_{2}} s_{i_{2}}^{2} \psi_{2} \\
\vdots \\
\sum_{j_{n}+i_{n}=1}\left(w_{0}^{n}\right)^{j_{n}}\left(w_{1}^{n}\right)^{i_{n}} s_{i_{n}}^{n} \psi_{n}
\end{array}\right]^{T}
$$

being $P$ a constant, symmetrical matrix with null diagonal in the said reference.

Expression (10) was proved path-independent (proving that $\partial f_{i} / \partial \psi_{j}=$ $\left.p_{i j}=p_{j i}=\partial f_{j} / \partial \psi_{i}\right)$, so the integral is identical for any $\Gamma$. Choosing the particular path formed by the 1-dimensional segments going from $(0, \ldots, 0)$ to $\left(x_{1}, 0, \ldots, 0\right)$, then to $\left(x_{1}, x_{2}, 0, \ldots\right)$, then to $\left(x_{1}, x_{2}, x_{3}, 0, \ldots\right)$ and so on until $\left(x_{1}, \ldots, x_{n}\right)$ is reached 1 , the integral (10) results in the actual explicit expression:

$$
V(x)=x^{T} P x+\sum_{k=1}^{n} \int_{0}^{x_{k}} \sum_{j_{k}+i_{k}=1}\left(w_{0}^{k}(\psi)\right)^{j_{k}}\left(w_{1}^{k}(\psi)\right)^{i_{k}} s_{i_{k}}^{k} \psi d \psi,
$$

where $\psi \in \mathbb{R}$ is now a one-dimensional dummy variable and $P$ is a matrix (without loss of generality, with null diagonal). Conversely, its gradient $\nabla V(x)$ is $f(x)$ being $f(\cdot)$ defined in (11). Such a fact can be proven from path-independence considerations, as originally done in [13], or, alternatively, by explicitly carrying out the straightforward differentiation of (12).

Then, a reformulation of the main result of [13], adapted to our notation, is the following theorem:

Theorem 1. The system (5) with $w_{j}^{k}\left(x_{k}(t)\right), k \in\{1,2, \ldots, n\}$ is asymptotically stable if the following conditions hold:

$$
\begin{gathered}
x^{T} P x+\sum_{k=1}^{n}\left(s_{j_{k}}^{k}-\varepsilon\right) x_{k}^{2} \geq 0 \\
-\sum_{\mathbf{k}_{0}+\mathbf{k}_{1}=\mathbf{2}} \mathbf{w}_{0}^{\mathbf{k}_{0}} \mathbf{w}_{1}^{\mathbf{k}_{1}} \sum_{\mathbf{i}+\mathbf{j}=\mathbf{k}_{1}, \mathbf{i} \leq \mathbf{1}, \mathbf{j} \leq \mathbf{1}} x^{T}\left(\bar{P}_{\mathbf{j}} A_{\mathbf{i}}+A_{\mathbf{i}}^{T} \bar{P}_{\mathbf{j}}+\varepsilon I\right) x \geq 0 .
\end{gathered}
$$

where $\bar{P}_{\mathbf{j}}=P+\operatorname{diag}\left(s_{1 j_{1}}, s_{2 j_{2}}, \ldots, s_{n j_{n}}\right)$ and $\varepsilon$ is a small positive constant.

\footnotetext{
${ }^{1}$ or, evidently, any other path, if desired.
} 
The reader is referred to the cited references for further details and proofs of the above-presented results. Trivially, by removing $x^{T}$ and $x$ and using Polya relaxations [22], the above scalar inequalities get converted into standard LMIs:

$$
\begin{gathered}
\bar{P}_{\mathbf{k}}-\varepsilon I \geq 0 \quad \forall \mathbf{k} \leq \mathbf{1} \\
\sum_{\mathbf{i}+\mathbf{j}=\mathbf{k}, \mathbf{i} \leq \mathbf{1}, \mathbf{j} \leq \mathbf{1}} \bar{P}_{\mathbf{j}} A_{\mathbf{i}}+A_{\mathbf{i}}^{T} \bar{P}_{\mathbf{j}}+\varepsilon I \leq 0 \quad \forall \mathbf{k} \leq \mathbf{2} .
\end{gathered}
$$

\subsection{Polynomial fuzzy models}

Consider now a more general case where $\tilde{h}$ in $(2)$ is a polynomial in nonlinearities $\eta$, say, of degree $c_{k}$ in $\eta_{k}$ for $k=1, \ldots q$. Then, the model can be expressed as the multi-dimensional TS one below where memberships have degree greater than 1 in the summations:

$$
\dot{x}(t)=\sum_{\mathbf{j}+\mathbf{i}=\mathbf{c}} n_{\mathbf{i}}^{\mathbf{c}} \mathbf{w}_{0}^{\mathbf{j}} \mathbf{w}_{1}^{\mathbf{i}} A_{\mathbf{i}} x(t),
$$

being $\mathbf{c}:=\left(c_{1}, c_{2}, \ldots, c_{q}\right)$ a degree vector where $c_{k}, \underset{\tilde{h}}{k}=\{1,2, \ldots, q\}$ is the degree each nonlinearity $\eta$ has in the polynomial $\tilde{h}$ in (2), and $n_{\mathbf{i}}^{\mathbf{c}}:=$ $\prod_{k=1}^{q} \frac{c_{k} !}{i_{k} !\left(c_{k}-i_{k}\right) !}$. The combinatorial number $n_{\mathbf{i}}^{\mathbf{c}}$ is the number of similar terms sharing a specific combination $\mathbf{w}_{0}^{\mathbf{j}} \mathbf{w}_{1}^{\mathbf{i}}$, which allows writing $\sum_{\mathbf{j}+\mathbf{i}=\mathbf{c}} n_{\mathbf{i}}^{\mathbf{c}} \mathbf{w}_{0}^{\mathbf{j}} \mathbf{w}_{1}^{\mathbf{i}}=1$, a property that proves to be useful in the quest for less conservative conditions derived from convex sums [34]. Note that the previously-considered tensor-product TS case in (2) is the particular case of $\mathbf{c}=(1, \ldots, 1)$.

Actually, if $\tilde{h}$ were a polynomial in both $\eta$ and $x$, then a so-called fuzzypolynomial mode ${ }^{2}$ would have been obtained in the form:

$$
\dot{x}(t)=\sum_{\mathbf{j}+\mathbf{i}=\mathbf{c}} n_{\mathbf{i}}^{\mathbf{c}} \mathbf{w}_{0}^{\mathbf{j}} \mathbf{w}_{1}^{\mathbf{i}} F_{\mathbf{i}}(x(t)):=F_{w}(x(t)),
$$

where $F_{\mathbf{i}}(x(t))$ are vertex polynomial models [5]. These general fuzzy polynomial models will, thus, be the subject of inquiry in the sequel.

The sum-of-squares (SOS) paradigm is widely used to prove stability of the above models. Indeed, a polynomial $p(x)$ is SOS (to be denoted by

\footnotetext{
${ }^{2}$ As discussed in [5], if $h$ in (1) is of class $\mathcal{C}^{p}$, a Taylor-series argumentation can prove the existence of such a fuzzy-polynomial model of degree $p$.
} 
$\left.p(x) \in \Sigma_{x}\right)$ if it can be decomposed as $\zeta^{T}(x) \Gamma \zeta(x)$ where $\zeta(x)$ is a vector of monomials and the so-called Gram-matrix $\Gamma$ is a positive semi-definite matrix, $\Gamma \geq 0$. Obviously, all SOS polynomials are non-negative, although the converse is not true [35].

Theorem 2 ([5, 36, 18]). The polynomial fuzzy model (18) is asymptotically stable if a polynomial Lyapunov function $V(x)=P(x)$ can be found verifying

$$
\begin{gathered}
P(x)-\varepsilon(x) \in \Sigma_{x}, \\
-\nabla P(x) F_{\mathbf{i}}(x)-\varepsilon(x) \in \Sigma_{x}, \quad \forall \mathbf{i} \leq \mathbf{c},
\end{gathered}
$$

where $\varepsilon(x)$ is a radially unbounded positive polynomial.

For a high-enough degree of $P(x)$ and $F_{\mathbf{i}}(x)$ if the nonlinear system admits a smooth Lyapunov function, the polynomial approach will eventually succeed, up to the gap of positive polynomials which are not SOS [35], if sufficient computational resources were available.

\section{Fuzzy-polynomial Lyapunov functions}

In [19], a fuzzy-polynomial LF was proposed $P_{w}(x)$, improving over Theorem 2 due to its larger representation capabilities. However, there was the

need of explicitly bounding $\frac{\partial w}{\partial x}$ by, for instance, other polynomials of the state (the authors proposed carrying out a fuzzy-polynomial model of the mentioned partial derivatives). This is an extension of the idea of bounding the value or $\dot{w}$ in [30] or bounding the gradient of the membership functions in [33]. Notwithstanding, as the goal of this work is enhancing the integral terms in Lyapunov functions, no further discussion of gradient/timederivative bounding will be considered in the sequel (actually, combination of approaches is possible, see discussion in Section 5).

\subsection{Problem statement}

The objective of this paper is generalising the LI Lyapunov function proposal in [13, 21] to a class of fuzzy-polynomial models in the form (18). Specifically, we will assume that the nonlinear model, written as expression (2), has the particular form:

$$
\dot{x}(t)=\tilde{h}\left(\eta_{1}\left(\rho_{1}(x)\right), \ldots, \eta_{q}\left(\rho_{q}(x)\right), x\right)
$$

where $\tilde{h}$ is a polynomial in its arguments $(\eta, x)$, with each $\eta_{j}: \mathbb{R} \mapsto \mathbb{R}$ being a real function of one variable, and being $\rho_{j}: \mathbb{R}^{n} \mapsto \mathbb{R}$ the argument 
to $\eta_{j}$; furthermore, $\rho_{j}(x)$ which will be assumed to be a polynomial in the state. Then, sector-nonlinearity modelling of $\eta_{j}$ allows building membership functions in (18) which depend on $\rho_{j}(x)$ :

$$
w_{0}^{j}\left(\rho_{j}\right)=\frac{\bar{\eta}_{j}-\eta_{j}\left(\rho_{j}\right)}{\bar{\eta}_{j}-\underline{\eta}_{j}}, w_{1}^{j}\left(\rho_{j}\right)=1-w_{0}^{j}\left(\rho_{j}\right), j \in\{1,2, \ldots, q\} .
$$

Thus, in the case under study, we will consider $w_{i}^{j}: \mathbb{R} \mapsto \mathbb{R}$, having the polynomial $\rho_{i}$ as argument, instead of the "generic" dependence $w_{i}^{j}(x)$ considered in the original expression (4). Actually, it can be easily shown that the cases in [13, 21] are a particular case of the above setup, details left to the reader. For instance, in [13], condition $\rho_{i} \equiv x_{i}$ was needed, as well as $q \leq n$. These assumptions are no longer needed in the present work, as discussed below.

The main goal of this paper is generalising $\rho_{i}$ to arbitrary polynomials, and to also consider the case in which the number of nonlinearities $q$ can be larger than the system's order $n$. Given that polynomials appear, the generalisation of [13] to the polynomial case (from LMI to SOS) comes as a side result but, importantly, advantages of the ideas here proposed can be achieved even in an LMI-only setup, as discussed in our conference paper [21. Hence, the LMIs in the cited works will be a particular case of our SOS approach.

Note that we do not need to model the gradient of the memberships because of the integral nature of the LF (following the main idea in the seminal work [13]), thus obtaining simpler conditions than [19] (which require such gradient model), but more powerful than standard SOS conditions (Theorem 2), due to the incorporation of $w(\cdot)$ in the LI Lyapunov function.

Example 1 (continued). Considering the model in (7), the approach in [13 cannot be "directly" applied to the above model using all three weighting functions: Theorem 1 can be applied by considering only fuzziness in the WFs $w_{j}^{1}(\cdot)$ in the Lyapunov function 12 , because it is the only one which depends on exactly a single state variable. Thus, Theorem 1 can consider the following integral form for $V(x)$ :

$$
V(x)=x^{T} P x+\int_{0}^{x_{2}} \sum_{i+j=1}\left(w_{0}^{1}(\psi)\right)^{i}\left(w_{1}^{1}(\psi)\right)^{j} s_{j 1} \psi d \psi .
$$

The above example motivates the need for extending the Lyapunov function with further integral terms depending on $w_{0}^{2}, w_{1}^{2}, w_{0}^{3}$ and $w_{1}^{3}$, to be dealt 
with in our proposals in next section. Note that a fuzzy-polynomial model (18) may be obtained for the model in example 11. if so wished; anyway, as the goal of this paper is comparing the flexibility of the more general Lyapunov function proposals, we intentionally restrain ourselves to just the TS model (7) in the later numerical computations over the nonlinear system in this example, in order to suitably compare with prior literature; such further improvements from more general polynomial modelling are left to the reader.

\section{Main Result}

Let us first consider a generic integral expression, motivated by (12), in the form:

$$
\bar{V}(\mu, \lambda):=P(\mu, \lambda)+\sum_{k=1}^{q} \int_{0}^{\lambda_{k}} \pi_{w}^{[k]}(\mu, \psi) d \psi
$$

where $\lambda \in \mathbb{R}^{q}, \mu \in \mathbb{R}^{s}$, for some $s$ to be later specified, are symbolic arguments (which will be later on replaced by state-dependent expressions), $P(\mu, \lambda)$ is an arbitrary polynomial function (depending on some decision variables), $\psi \in \mathbb{R}$ is a uni-dimensional dummy integral variable, and $\pi_{w}^{[k]}(\mu, \psi): \mathbb{R}^{s+1} \mapsto \mathbb{R}$ are given by the fuzzy summations

$$
\pi_{w}^{[k]}(\mu, \psi)=\sum_{s_{k}+l_{k}=d_{k}} n_{l_{k}}^{d_{k}}\left(w_{0}^{k}(\psi)\right)^{s_{k}}\left(w_{1}^{k}(\psi)\right)^{l_{k}} s_{l_{k}}^{k}(\mu, \psi)
$$

where $d_{k}$ is a Polya complexity parameter [34], $n_{l_{k}}^{d_{k}}$ is a combinatorial number $n_{l_{k}}^{d_{k}}=\frac{d_{k} !}{l_{k} !\left(d_{k}-l_{k}\right) !}$, and $s_{l_{k}}^{k}(\mu, \psi)$ is a polynomial parameterised, too, with some decision variables. As before, the Polya complexity parameters will be arranged into a "Polya degree vector", to be denoted as $\mathbf{d}:=\left(d_{1}, \ldots, d_{q}\right) \in$ $\mathbb{N}^{q}$.

In order to be used as a Lyapunov function, the gradient of $\bar{V}$ needs to be computed. Instead of line-integral argumentations, we will use explicit differentiation, as justified earlier on. Thus, the components of the gradient of $\bar{V}$ are given by:

$$
\frac{\partial \bar{V}}{\partial \mu_{i}}=\frac{\partial P}{\partial \mu_{i}}(\mu, \lambda)+\sum_{k=1}^{q} \int_{0}^{\lambda_{k}} \frac{\partial \pi_{w}^{[k]}}{\partial \mu_{i}}(\mu, \psi) d \psi
$$


and

$$
\frac{\partial \bar{V}}{\partial \lambda_{k}}=\frac{\partial P}{\partial \lambda_{k}}(\mu, \lambda)+\pi_{w}^{[k]}\left(\mu, \lambda_{k}\right)
$$

The above structure (24) will be used to build Lyapunov functions in Section 3.1, once relevant positiveness conditions formulated below do hold.

Theorem 3. If $P(\mu, \lambda) \in \Sigma_{\mu, \lambda}$ and $s_{l_{k}}^{k}(\mu, \psi) \psi \in \Sigma_{\mu, \psi}$, for all $0 \leq l_{k} \leq d_{k}$, then $\bar{V}(\mu, \lambda) \geq 0$.

Proof. Condition $s_{l_{k}}^{k}(\mu, \psi) \psi \in \Sigma_{\mu, \psi}$ implies that $s_{l_{k}}^{k}$ has the same sign as $\psi$. As $\pi_{w}^{[k]}$ is a sum of $s_{l_{k}}^{k}$ multiplied by positive coefficients, we can assert that $\pi_{w}^{[k]}(\mu, \psi) \psi \geq 0$ and, for any $\tau>0$, we have $\pi_{w}^{[k]}(\mu, \psi) \psi / \tau \geq 0$. Hence,

$$
\int_{0}^{\lambda_{k}} \pi_{w}^{[k]}(\mu, \psi) d \psi=\lim _{h \rightarrow 0^{+}} \int_{h}^{1} \pi_{w}^{[k]}\left(\mu, \tau \lambda_{k}\right) \lambda_{k} d \tau \geq 0
$$

where the rightmost integral comes from the change $\psi=\tau \lambda_{k}$, hence $\tau$ should range from zero to 1 , and the last inequality comes from the fact that $\pi_{w}^{[k]}\left(\mu, \tau \lambda_{k}\right) \lambda_{k}=\pi_{w}^{[k]}(\mu, \psi) \psi / \tau \geq 0$. Note that the limit in the above expression exists from continuity of $\pi_{w}^{[k]}$. Therefore, $\bar{V}$ is expressed as the sum of two non-negative quantities if conditions in the theorem statement hold.

The above theorem can be made less conservative, introducing some additional decision variables (non-fuzzy polynomials $\underline{s}_{k}$ ) which "link" the nonintegral and integral parts, as follows:

Theorem 4 . If there exist polynomials $\underline{s}_{k}(\mu, \psi)$, for $k \in\{1,2, \ldots, q\}$, such that

$$
\left(s_{l_{k}}^{k}(\mu, \psi)-\underline{s}_{k}(\mu, \psi)\right) \psi \in \Sigma_{\mu, \psi}
$$

and

$$
V_{1}(\mu, \lambda):=P(\mu, \lambda)+\sum_{k=1}^{q} \int_{0}^{\lambda_{k}} \underline{s}_{k}(\mu, \psi) d \psi \in \Sigma_{\mu, \lambda}
$$

then $\bar{V}(\mu, \lambda) \geq 0$ for all $\mu, \lambda$.

Proof. We can express:

$$
\bar{V}(\mu, \lambda)=V_{1}(\mu, \lambda)+V_{2}(\mu, \lambda)
$$


where $V_{1}$ is the polynomial defined in 29 and

$$
V_{2}(\mu, \lambda):=\sum_{k=1}^{q} \int_{0}^{\lambda_{k}}\left(\pi_{w}^{[k]}(\psi)-\underline{s}_{k}(\mu, \psi)\right) d \psi
$$

and Theorem 3 can now be applied changing the original $\bar{V}(\cdot)$ by $V_{1}(\cdot)$, and changing $s_{l_{k}}^{k}$ in the referred theorem for $s_{l_{k}}^{k}-\underline{s}_{k}$, as stated in (28).

Next section will apply the above results to building Lyapunov functions. In order to avoid integral terms in the gradient of $V$, the restriction $s_{l_{k}}^{k}(\mu, \psi)$ being only dependent on $\psi$ will be enforced in the sequel, i.e., we will only consider $s_{l_{k}}^{k}(\psi)$.

\subsection{Stability}

Consider now a Lyapunov function, using the structure (24), defined as:

$$
V(x):=\bar{V}(E x, \rho(x))=P(E x, \rho(x))+\sum_{k=1}^{q} \int_{0}^{\rho_{k}} \pi_{w}^{[k]}(\psi) d \psi
$$

where $E x$ selects only the components of the state which do not explicitly appear in $\rho(x)$ (thus, avoiding repeated arguments): for instance, in the original setting in [13], $E$ would be zero as $\rho(x) \equiv x$; in the 2nd-order system in Example 1, we would set $E:=(10)$, so $E x=x_{1}$ because $\rho_{1}(x)=x_{2}$.

Using positiveness results in Theorem 4 and adding derivative-related decrescence conditions allows to state the main result below:

Theorem 5. Consider a polynomial fuzzy model (18), with degree vector c, with the membership function structure $w_{j}^{k}\left(\rho_{k}(x)\right)$, arising from (21) and (22). Consider, too, a given degree vector $\mathbf{d}=\left(d_{1}, d_{2}, \ldots, d_{q}\right)$, see (25), and the Lyapunov function structure (32) and an arbitrary radially unbounded polynomial $\varepsilon(x)$, such that $\varepsilon(0)=0$ and $\varepsilon(x)>0$ elsewhere. Then, the origin $x(t)=0$ of such system is asymptotically stable if there exist polynomial functions $P(E x, \rho(x)), s_{l_{k}}^{k}(\psi)$, and $\underline{s}_{k}(\psi)$, such that the following SOS 
conditions hold for all $0 \leq l_{k} \leq d_{k}, 0 \leq b_{j} \leq e_{j}, j, k \in\{1,2, \ldots, q\}$ :

$$
\begin{gathered}
\left(s_{l_{k}}^{k}(\psi)-\underline{s}_{k}(\psi)\right) \psi \in \Sigma_{\psi} \\
P(E x, \rho(x))+\sum_{k=1}^{q} \int_{0}^{\rho_{k}} \underline{s}_{k}(\psi) d \psi-\varepsilon(x) \in \Sigma_{x} \\
-\sum_{l_{j}+i_{j}=b_{j}} n_{\mathbf{l}}^{\mathbf{d}} n_{\mathbf{i}}^{\mathbf{c}}(\nabla P(E x, \rho(x))[E
\end{gathered}
$$

where $\mathbf{e}=\left(c_{1}+d_{1}, c_{2}+d_{2}, \ldots, c_{q}+d_{q}\right)$.

Proof. Conditions (33) and (34) are the translation ${ }^{3}$ to the current notation of conditions (28) and (29). Thus, application of Theorem 4 ensures that $V(x)$ in 32 fulfills $V(x) \geq \varepsilon(x)$.

Now, the derivative of the Lyapunov function can be expressed as:

$$
\dot{\bar{V}}(\mu, \lambda)=\left(\frac{\partial \bar{V}}{\partial \mu} \frac{\partial \mu}{\partial x}+\frac{\partial \bar{V}}{\partial \lambda} \frac{\partial \lambda}{\partial x}\right) \cdot \dot{x}(t)
$$

so, with the choice of arguments to $\bar{V}(\cdot)$ being $\mu:=E x$ and $\lambda:=\rho(x)$, we have that the time derivative above (corresponding to the time derivative of (32)) becomes:

$$
\begin{aligned}
& \dot{V}(x)=\nabla P(E x, \rho(x))[E \nabla \rho] \dot{x}(t) \\
& +\left[\pi_{w}^{[1]}\left(\rho_{1}(x)\right) \quad \cdots \quad \pi_{w}^{[q]}\left(\rho_{q}(x)\right)\right] \nabla \rho(x) \dot{x}(t) \leq 0 .
\end{aligned}
$$

Replacing $\dot{x}(t)$ by its model (18), and $\pi_{w}^{[k]}$ by its definition (25), we get:

$$
\begin{aligned}
& \sum_{\mathbf{j}+\mathbf{i}=\mathbf{c}} n_{\mathbf{i}}^{\mathbf{c}} \mathbf{w}_{0}^{\mathbf{j}} \mathbf{w}_{1}^{\mathbf{i}} \nabla P(E x, \rho(x))[E \nabla \rho] F_{\mathbf{i}}(x) \\
& +\sum_{k=1}^{q} \sum_{s_{k}+l_{k}=d_{k}}\left(w_{0}^{k}\left(\rho_{k}\right)\right)^{s}\left(w_{1}^{k}\left(\rho_{k}\right)\right)^{l} n_{l}^{d_{k}} s_{l_{k}}^{k}\left(\rho_{k}\right) \nabla \rho_{k} \sum_{\mathbf{j}+\mathbf{i}=\mathbf{c}} n_{\mathbf{i}}^{\mathbf{c}} \mathbf{w}_{0}^{\mathbf{j}} \mathbf{w}_{1}^{\mathbf{i}} F_{\mathbf{i}}(x) \leq 0,
\end{aligned}
$$

\footnotetext{
${ }^{3}$ Actually, note that $(29)$ poses SOS conditions on two variables $(\mu, \lambda)$ so applicability of Theorem 4 would hold even if the explicit relationship between these variables were unknown. However, as $\rho_{i}$ are known polynomials in (32), substitution of these polynomials by their explicit expressions renders an easier SOS problem only in variables $x$ in (34).
} 
which is equivalent to the homogeneous summation of degree vector $\mathbf{e}:=$ $\left(c_{1}+d_{1}, c_{2}+d_{2}, \ldots, c_{q}+d_{q}\right)$ below:

$$
\begin{gathered}
\sum_{\mathbf{a}+\mathbf{b}=\mathbf{e}} \mathbf{w}_{0}^{\mathbf{a}} \mathbf{w}_{1}^{\mathbf{b}} \sum_{l_{j}+i_{j}=b_{j}} n_{\mathbf{1}}^{\mathbf{d}} n_{\mathbf{i}}^{\mathbf{c}}\left(\nabla P(E x, \rho(x))[E \nabla \rho] F_{\mathbf{i}}\right. \\
\left.+\left(\sum_{k=1}^{q} s_{l_{k}}^{k}\left(\rho_{k}\right) \nabla \rho_{k}\right) F_{\mathbf{i}}(x)\right)+\varepsilon(x) \leq 0,
\end{gathered}
$$

Carrying out fuzzy-summation manipulations as to isolate each of the summation coefficients, we get the sufficient condition (35), which guarantees $\dot{V}(x)<0$, thus concluding the proof.

Note that Polya relaxations of the fuzzy summations (35) may be carried out to further reduce conservatism, but details on them are omitted for brevity.

In the particular case where $\rho_{k}(x)$ is an arbitrary linear function of the state $x(t)$, i.e., $\rho_{k}(x)=l_{1}^{k} x_{1}(t)+l_{2}^{k} x_{2}(t)+\cdots+l_{n}^{k} x_{n}(t)=L^{[k]} x(t), \forall k \in$ $\{1,2, \ldots, q\}$, if the Lyapunov function is also chosen to be quadratic, then Theorem 5 reduces to the stability conditions in [21, Thm. 4], as stated next: Corollary 1. The origin $x(t)=0$ of the TS model (17) with the membership function structure $w_{j}^{k}\left(\rho_{k}(x)\right)$ and $\rho_{k}(x)$ being an arbitrary linear function of the state $x(t)$, i.e., $\rho_{k}(x)=l_{1}^{k} x_{1}(t)+l_{2}^{k} x_{2}(t)+\cdots+l_{n}^{k} x_{n}(t)=L^{[k]} x(t)$, $\forall k \in\{1,2, \ldots, q\}$, is asymptotically stable if the following conditions hold:

$$
\begin{gathered}
x^{T} P x+\sum_{k=1}^{q} s_{j_{k}}^{k} x^{T}\left(L^{[k]}\right)^{T} L^{[k]} x(t)-\varepsilon x^{T} x \geq 0, \forall j_{k} \in\{0,1\} \\
-\sum_{\mathbf{k}_{0}+\mathbf{k}_{1}=\mathbf{2}} \mathbf{w}_{0}^{\mathbf{k}_{0}} \mathbf{w}_{1}^{\mathbf{k}_{1}} \sum_{\mathbf{i}+\mathbf{j}=\mathbf{k}_{1}, \mathbf{i} \leq \mathbf{1}, \mathbf{j} \leq \mathbf{1}} x^{T}\left(\bar{P}_{\mathbf{j}} A_{\mathbf{i}}+A_{\mathbf{i}}^{T} \bar{P}_{\mathbf{j}}+\varepsilon I\right) x \geq 0,
\end{gathered}
$$

where $\bar{P}_{\mathbf{j}}=P+\operatorname{diag}\left(s_{j_{1}}^{1}, s_{j_{2}}^{2}, \ldots, s_{j_{n}}^{n}\right)$, being $P=P^{T} \in \mathbb{R}^{n \times n}$ with null diagonal, and $\varepsilon$ is a small positive constant. Obviously the above quadratic SOS conditions can be, trivially, considered to be an LM!

Proof. Considering the Lyapunov function candidate 32 with

$$
P(E x, \rho(x))=x^{T} P x,
$$

\footnotetext{
${ }^{4}$ See conditions (11) and (12) in [21].
} 
$P$ defined as above with null diagonal, and

$$
\pi_{w}^{[k]}(\psi)=2 \sum_{s_{k}+l_{k}=1}\left(w_{0}^{k}(\psi)\right)^{s_{k}}\left(w_{1}^{k}(\psi)\right)^{l_{k}} s_{l_{k}}^{k} \psi
$$

Rewriting (32), we get the following function:

$$
V(x)=x^{T} P x+\sum_{k=1}^{q} \int_{0}^{L^{[k]} x_{k}} \pi_{w}^{[k]}(\psi) d \psi .
$$

By Theorem 4, expression (38) is positive if there exists $\underline{s}_{k}$ such that:

$$
\begin{aligned}
& p_{l_{k}}^{k} \psi^{2}-\underline{s}_{k} \psi^{2} \in \Sigma_{\psi} \\
& x^{T} P x+\sum_{k=1}^{q} \int_{0}^{L^{[k]} x_{k}} \underline{s}_{k} \psi d \psi \\
& =x^{T} P x+\sum_{k=1}^{q} \underline{s}_{k} x^{T}\left(L^{[k]}\right)^{T} L^{[k]} x(t)-\varepsilon x^{T} x \in \Sigma_{x} .
\end{aligned}
$$

Since $P$ is null diagonal, setting $\underline{s}_{k}=\min \left(s_{0}^{k}, s_{1}^{k}\right)$, then (36) implies the previous condition.

The following condition on the time derivative of the Lyapunov function 38

$$
\dot{V}(x)=x^{T}\left(P A_{w}+A_{w}^{T} P\right) x+2 \sum_{k=1}^{q} \pi_{w}^{[k]}(x) A_{w} x<0,
$$

is equivalent to that in (37) as can be seen performing similar steps as those in proof of Theorem 5 .

\section{Examples}

Example 1 (continued). The motivating example considering the model in (7) will be now numerically solved with the proposed results, and compared with alternative prior approaches. In particular, stability of the system (7) will be studied for different values of constant parameters $a \in[10,13]$ and $b \in[50,60]$.

First, recall that the results in [13], i.e., Theorem 1] can be applied only with the Lyapunov function (23), with integral terms only depending on $x_{2}$, as previously discussed on page 10 . 


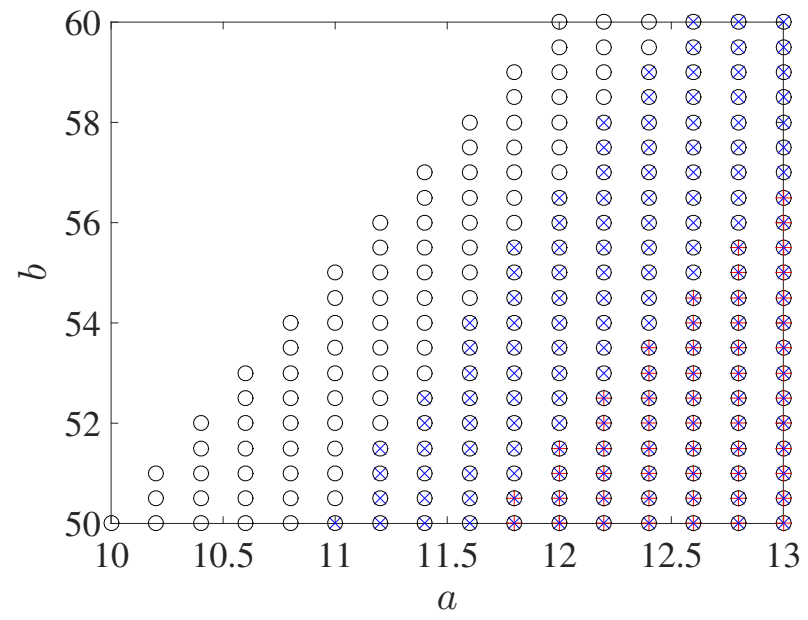

Figure 1: Feasibility sets for Example 1: (o) for Theorem 5: $(\times)$ for Theorem 1 $(+)$ for Quadratic Lyapunov function.

However, our proposal in Theorem 5 can consider all three nonlinearities. If we apply Theorem 5 with $\varepsilon(x)=10^{-4} x^{T} x$ and the following Polynomial Line-integral Lyapunov function

$$
V(x)=p_{1} x_{1}^{2}+p_{2} x_{1} x_{2}+p_{3} x_{2}^{2}+\sum_{k=1}^{3} \int_{0}^{\rho_{k}}\left(w_{0}^{k}(\psi) s_{0 k} \psi+w_{1}^{k}(\psi) s_{1 k} \psi\right) d \psi
$$

where $p_{1}, p_{2}, p_{3}$, and $s_{j k}$ are decision variables, the obtained feasible set of solutions is marked with (o) in Figure 1, within the ranges of $a$ and $b$ above mentioned. For the sake the comparison, in Figure 1 the feasible set of solutions obtained if the classical quadratic approach $V=x^{T} P x$ is applied is marked with a $(+)$; last, $(\times)$ points out the feasible set of solutions obtained if the approach in [13] is applied considering only the WFs $w_{j}^{1}(\cdot)$ in the Lyapunov function (23) with, too $\varepsilon=10^{-4}$. As expected, [13] improves over the plain quadratic case, but our new approach produces the largest feasible set of solution: $5^{5}$ due to the two additional integral terms apart from the one in $(23)$.

\footnotetext{
${ }^{5}$ Note that, although this example has detailed the developments for polynomial arguments to $\rho$, similar improvements occur even if the arguments of $\rho$ were just linear functions, as discussed earlier in this work (Corollary 1 , taken from our conference paper [21]).
} 
Example 2. In this example, we will compare our proposal with a "standard" sum-of-squares approach (recalled here as Theorem 2), i.e., with a polynomial non-fuzzy Lyapunov function (without integral terms). In order to carry out such a comparison, we will consider the following nonlinear model:

$$
\begin{aligned}
& \dot{x}_{1}=x_{2} \\
& \dot{x}_{2}=-2 x_{1}-x_{2}-0.5 \kappa x_{1}\left(1+\sin \left(\rho_{1}(x)\right)\right),
\end{aligned}
$$

where $\rho_{1}(x)=-4 x_{2}-5 x_{2} x_{1}+x_{1}^{2}-2 x_{2}^{2}$ and $\kappa$ is a non-negative parameter, so the objective is finding the largest possible $\kappa$ such that several sets of SOS conditions (corresponding to different LF proposals) render feasible, to compare them. Applying the sector nonlinearity approach to $\sin \left(\rho_{1}(x)\right)$, we obtained the following TS model:

$$
\dot{x}=\sum_{i_{1}=0}^{1} w_{i_{1}}^{1}\left(\rho_{1}\right) A_{i_{1}} x,
$$

where

$$
A_{0}=\left[\begin{array}{cc}
0 & 1 \\
-2 & -1
\end{array}\right], \quad A_{1}=\left[\begin{array}{cc}
0 & 1 \\
-2-\kappa & -1
\end{array}\right],
$$

and $w_{0}^{1}\left(\rho_{1}\right)=0.5\left(1-\sin \left(\rho_{1}\right)\right), w_{1}^{1}\left(\rho_{1}\right)=1-w_{0}^{1}\left(\rho_{1}\right)$.

Note that, as $\rho_{1}$ is neither a state nor a linear function of the state variables, integral LF terms from the proposals in [13] or [21] cannot be applied.

Following our approach in this work, if Theorem 5 is applied with $\mathbf{d}=(1)$, $\varepsilon(x)=10^{-4}\left(x_{1}^{2}+x_{2}^{2}\right)$, and the following Polynomial Line-Integral Lyapunov function, which incorporates degree- 4 monomials:

$$
\begin{aligned}
& V(x)=p_{1} x_{1}^{2}+p_{2} x_{1} x_{2}+p_{3} x_{2}^{2}+p_{4} x_{1}^{3}+p_{5} x_{1}^{2} x_{2}+p_{6} x_{1} x_{2}^{2}+p_{7} x_{2}^{3}+p_{8} x_{1}^{4} \\
& \quad+p_{9} x_{1}^{3} x_{2}+p_{10} x_{1}^{2} x_{2}^{2}+p_{11} x_{1} x_{2}^{3}+p_{12} x_{2}^{4}+\int_{0}^{\rho_{1}}\left(w_{0}^{1}(\psi) s_{01} \psi+w_{1}^{1}(\psi) s_{11} \psi\right) d \psi
\end{aligned}
$$

such that $\left(s_{01} \psi-\underline{s}_{1} \psi\right) \psi \in \Sigma_{\psi}$, where $p_{i}, i \in\{1,2, \ldots, 12\}, \underline{s}_{1}, s_{11}$ and $s_{01}$ are decision variables, our approach can guarantee stability for $\kappa=6.5046$. The resulting Lyapunov function for $\kappa=6.5046$ is

$$
\begin{aligned}
V(x) & =266.1084 x_{1}^{4}+91.8725 x_{1}^{3} x_{2}+54.6768 x_{1}^{3}+116.435 x_{1}^{2} x_{2}^{2}+10.7059 x_{1}^{2} x_{2} \\
& +76.9601 x_{1}^{2}+11.1967 x_{1} x_{2}^{3}-38.9486 x_{1} x_{2}^{2}+12.3850 x_{1} x_{2}+5.6442 x_{2}^{4} \\
& -20.2489 x_{2}^{3}-3.8261 x_{2}^{2}+\int_{0}^{\rho_{1}}\left(w_{0}^{1}(\psi) 3.2041 \psi+w_{1}^{1}(\psi) 1.9146 \psi\right) d \psi
\end{aligned}
$$




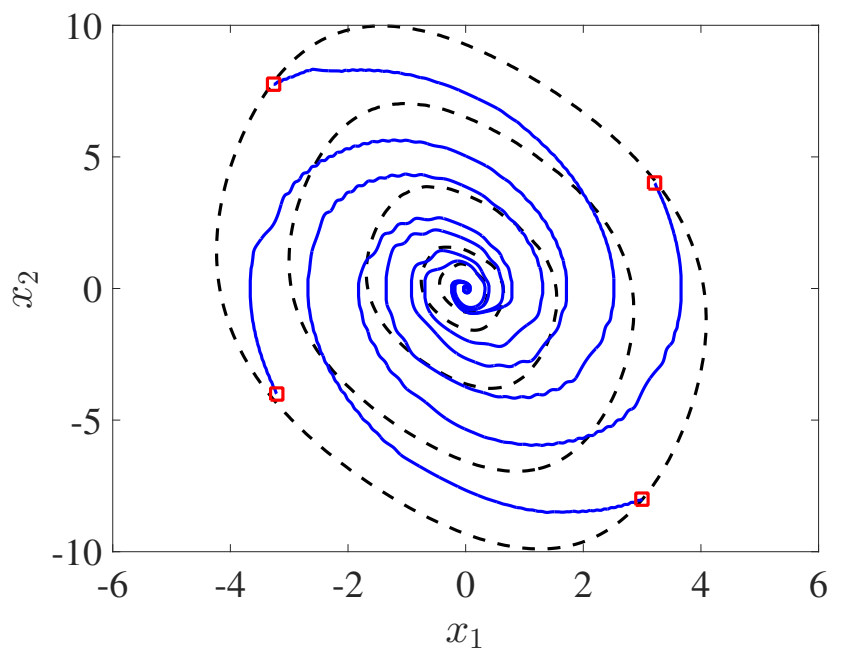

Figure 2: Lyapunov sets (dashed lines) and some trajectories (solid lines) for Example 2.

with $\underline{s}_{k}=1.391$. In Figure 2 some level sets of $V(x)$ and some system trajectories are shown for illustration purposes.

For the sake of comparison, Table 1 presents the maximum value of the parameter $\kappa$ keeping conditions in Theorem 5 feasible ${ }^{6}$ for several degrees of the classical polynomial LF component $P\left(E x, \rho_{1}(x)\right)$ (left column) and the integral ones (middle column) with $\mathbf{d}=(2)$. Thus, the standard SOS approach corresponds to the rows where $\operatorname{deg}\left(s_{l_{k}}^{k}(\psi)\right)$ is empty (labelled with a dash). For instance, a 4th-degree non-integral term plus a degree 1 integral term achieves better results than a non-integral LF of degree 12. From the numerical figures in the table, either increasing the non-integral polynomial degree or that of the integral term seem to improve results, however the incorporation of integral terms seems very effective with significantly less decision variables than the high-degree non-integral options, while achieving better performance.

For information, the used solver in the numerical examples in this paper was Mosek 7.1 [38], under the programming language YALMIP 20150919 [39], and running on Matlab R2015a with default tolerances.

\footnotetext{
${ }^{6}$ The function $\varepsilon(x)$ was chosen, following [37, as: $\varepsilon(x)=\sum_{i=1}^{n} \sum_{j=1}^{d} \epsilon_{i j} x_{i}^{2 j}$ where $d$ is the degree of $P(E x, \rho(x))$ and the $\epsilon$ 's satisfy $\sum_{j=1}^{d} \epsilon_{i j}>\gamma, \quad \forall i \in\{1,2, \ldots, n\}$ with $\gamma$ a positive number $\left(1 \times 10^{-4}\right)$, and $\epsilon_{i j} \geq 0$ for all $i$ and $j$.
} 
Table 1: Maximum $\kappa$ for polynomial line-integral LF with Theorem 5 , and standard SOS Theorem 2

\begin{tabular}{ccccc}
$\operatorname{deg}(P(E x, \rho(x)))$ & $\operatorname{deg}\left(s_{l_{k}}^{k}(\psi)\right)$ & $\kappa$ & $\begin{array}{c}\text { Decision } \\
\text { variables in } \\
V(x)\end{array}$ & $\begin{array}{c}\text { Average Solver } \\
\text { time (s) }\end{array}$ \\
\hline 2 & - & 3.8284 & 3 & 0.1660 \\
4 & - & 5.7393 & 12 & 0.1740 \\
8 & - & 6.3981 & 42 & 0.1960 \\
12 & - & 6.6537 & 88 & 0.3440 \\
4 & 1 & 7.0880 & 16 & 0.1840 \\
4 & 3 & 7.1018 & 24 & 0.1960 \\
8 & 1 & 7.2990 & 46 & 0.1940 \\
8 & 3 & 7.6879 & 54 & 0.2160 \\
12 & 1 & 8.3010 & 92 & 0.4760 \\
12 & 3 & 8.9234 & 100 & 0.4920 \\
\hline
\end{tabular}

\section{Discussion}

In this section, once the results and example have been presented, a brief discussion on the advantages provided and room for further enhancements will be provided next.

Regarding the chosen nonlinear model for the examples, note that they have been intentionally written as TS models in order to compare with prior literature, but other polynomial models for the same nonlinear systems may be amenable to our proposal (such as the Taylor-series approach [5]), details left to the reader.

Also, for simplicity, global bounds on the nonlinearities have been considered (they are trigonometric functions). Nevertheless, the approach would equally work on compact modelling regions where suitable bounds for $x$ and $\rho$ would be available. Obviously, the advantages of non-quadratic/fuzzyLF-SOS approaches would vanish for very small modelling regions, as the resulting model would equal the linearisation (in a TS case) or the truncated Taylor series (in the generic polynomial setup). Nevertheless, comparison of results with different sizes of modelling region has not been considered of interest, for brevity.

Apart from the concrete example, in a generic case, our approach has advantages if the nonlinearities can be expressed as a single-variable real function composed with a polynomial one; in this case, the polynomial nature 
of the arguments to nonlinearities is duly exploited. It would not apply to, for instance to $\rho(x)=\cos \left(e^{x_{1}}-\arctan x_{2}^{2}\right)$.

Note, too, that further relaxation of the result would be obtained by combining it with a variety of approaches in fuzzy control literature, which relax conservatism based on other ideas unrelated to our integral Lyapunov function proposal:

1. Increasing the degree of the polynomial term of the Lyapunov function $P(\cdot, \cdot)$ in 32 .

2. Get a less conservative model via increasing the degree of the polynomial consequents, [5].

3. Use a standard "fuzzy"-polynomial Lyapunov function in the nonintegral part of (32) replacing $P(E x, \rho)$ by $P(E x, \rho, \mathbf{w})$ with expressions similar to $(9)$, incorporating information on the time-derivatives or the gradient of the memberships [19].

4. Use other results depending on membership shape. For instance, in Example 1, based on the actual nonlinearities, we could assert expressions such as $\gamma(w):=\left(w_{0}^{1}\right)^{2}-0.5 w_{0}^{2} w_{0}^{3}-1 \leq 0$ or/and $\gamma(w):=$ $w_{0}^{2} w_{0}^{3}-\rho_{2}(x)^{2} \rho_{3}(x)^{2} \leq 0$, a restriction that can be included via a suitable Positivstellensatz multiplier $R(x, \rho) \gamma(w)$ in the SOS conditions [22, 40].

\section{Conclusion}

This paper presents a general SOS condition for the stability analysis of a class of nonlinear models via a polynomial Lyapunov function with integral terms which has been suitably parameterised. Compared to prior literature, two improvements are presented: first, the generalisation to a polynomial case of earlier LMI line-integral results; second, the new approach allows the line-integral approach to be applied to a larger class of TS models, where their WFs arguments can be arbitrary sets of polynomial functions of the system states, instead of only each of the states being the argument to a single WF considered in [13]. Unfortunately, as in the original reference, controller design problems cannot be cast as convex optimisation ones. 


\section{Acknowledgements}

The authors gratefully to the financial support of Spanish ministry of Economy and European Union, grant DPI2016-81002-R (AEI/FEDER, UE), the CONACyT/COECyT Sonora scholarship 383252, and Project ITSONPROFAPI-CA 2017-0088.

\section{References}

[1] T. Takagi, M. Sugeno, Fuzzy identification of systems and its applications to modeling and control, IEEE Transactions on Systems, Man and Cybernetics 15 (1) (1985) 116-132.

[2] J. S. Shamma, J. R. Cloutier, A linear parameter varying approach to gain scheduled missile autopilot design, in: American Control Conference, 1992, IEEE, 1992, pp. 1317-1321.

[3] T. Taniguchi, K. Tanaka, H. Wang, Model construction, rule reduction and robust compensation for generalized form of Takagi-Sugeno fuzzy systems, IEEE Transactions on Fuzzy Systems 9 (2) (2001) 525-537.

[4] K. Tanaka, H. Wang, Fuzzy Control Systems Design and Analysis. A linear matrix inequality approach, John Wiley and Sons, New York, 2001.

[5] A. Sala, C. Ariño, Polynomial fuzzy models for nonlinear control: a Taylor series approach, IEEE Transactions on Fuzzy Systems 17 (6) (2009) 1284-1295.

[6] G. Chesi, Estimating the domain of attraction for non-polynomial systems via LMI optimizations, Automatica 45 (6) (2009) 1536-1541.

[7] S. Boyd, L. Ghaoui, E. Feron, V. Belakrishnan, Linear Matrix Inequalities in System and Control Theory, Vol. 15, SIAM: Studies In Applied Mathematics, Philadelphia, USA, 1994.

[8] K. Tanaka, M. Sugeno, Stability analysis of fuzzy systems using Lyapunov's direct method, in: Proceedings of the North America Fuzzy Information Processing Society, Toronto, Canada, 1990, pp. 133-136. 
[9] M. Johansson, A. Rantzer, K. Arzen, Piecewise quadratic stability of fuzzy systems, IEEE Transactions on Fuzzy Systems 7 (6) (1999) 713722 .

[10] T. Gonzalez, A. Sala, M. Bernal, B. Aguiar, Piecewise-takagi-sugeno asymptotically exact estimation of the domain of attraction of nonlinear systems, Journal of the Franklin Institute 354 (3) (2017) 1514-1541.

[11] T. Guerra, L. Vermeiren, LMI-based relaxed non-quadratic stabilization conditions for nonlinear systems in Takagi-Sugeno's form, Automatica 40 (5) (2004) 823-829.

[12] T. Guerra, M. Bernal, Strategies to exploit non-quadratic local stability analysis, International Journal of Fuzzy Systems 14 (3) (2012) 372-379.

[13] B. Rhee, S. Won, A new fuzzy Lyapunov function approach for a TakagiSugeno fuzzy control system design, Fuzzy Sets and Systems 157 (9) (2006) 1211-1228.

[14] C. Ariño, , A. Sala, E. Pérez, B. F., A. Querol, Asymptotically exact stabilisation for constrained discrete Takagi-Sugeno systems via setinvariance, Fuzzy Sets and Systems 316 (2017) 117-138.

[15] F. Wu, S. Prajna, SOS-based solution approach to polynomial LPV system analysis and synthesis problems, International Journal of Control 78 (8) (2005) 600-611.

[16] K. Tanaka, H. Yoshida, H. Ohtake, H. Wang, A sum of squares approach to stability analysis of polynomial fuzzy systems, in: Proceedings of the 2007 American Control Conference, New York, USA, 2007, pp. 40714076 .

[17] S. Prajna, A. Papachristodoulou, P. Seiler, P. Parrilo, SOSTOOLS: Sum of squares optimization toolbox for MATLAB, [Online], available from http://www.cds.caltech.edu/sostools and http://www.mit. edu/parrilo/sostools (2004).

[18] S. Prajna, A. Papachristodoulou, P. Seiler, P. Parrilo, SOSTOOLS and its control applications, in: Positive polynomials in control, Vol. 312 of Lecture Notes in Control and Information Science, Springer, 2005, pp. $272-292$. 
[19] M. Bernal, A. Sala, A. Jaadari, G. T.M., Stability analysis of polynomial fuzzy models via polynomial fuzzy Lyapunov functions, Fuzzy Sets and Systems 185 (1) (2011) 5-14.

[20] Y. Chen, M. Tanaka, K. Tanaka, H. Wang, Stability analysis and regionof-attraction estimation using piecewise polynomial Lyapunov functions: Polynomial fuzzy model approach, IEEE Transactions on Fuzzy Systems 23 (4) (2015) 1314-1322.

[21] T. Gonzalez, A. Sala, M. Bernal, R. Robles, A generalisation of lineintegral Lyapunov function for Takagi-Sugeno systems, in: Proceedings of the 20th World Congress of the International Federation of Automatic Control, Toulouse, France, 2017.

[22] C. Ariño, A. Sala, Relaxed LMI conditions for closed-loop fuzzy systems with tensor-product structure, Engineering Applications of Artificial Intelligence 20 (8) (2007) 1036-1046.

[23] R. Marquez, T. Guerra, A. Kruszewski, M. Bernal, Improvements on non-quadratic stabilization of takagi-sugeno models via line-integral lyapunov functions, in: Proceedings of the 2013 IFAC International Conference on Intelligent Control and Automation Science, Chengdu, China, 2013, pp. 473-478.

[24] R. Marquez, T. Guerra, A. Kruszewski, M. Bernal, Non-quadratic stabilization of second order continuous takagi-sugeno descriptor systems via line-integral lyapunov function, in: Proceedings of the 2014 IEEE International Conference on Fuzzy Systems, Beijing, China, 2014, pp. $2451-2456$.

[25] R. Robles, A. Sala, M. Bernal, T. Gonzalez, Subspace-based TakagiSugeno modeling for improved LMI performance, IEEE Transactions on Fuzzy Systems 25 (4) (2017) 754-767.

[26] V. Campos, F. Souza, L. Torres, R. Palhares, New stability conditions based on piecewise fuzzy Lyapunov functions and tensor product transformations, IEEE Transactions on Fuzzy Systems 21 (4) (2013) 748-760.

[27] E. Tognetti, R. Oliveira, P. Peres, Selective $\mathcal{H}_{2}$ and $\mathcal{H}_{\infty}$ stabilization of Takagi-Sugeno fuzzy systems, IEEE Transactions on Fuzzy Systems 19 (5) (2011) 890-900. 
[28] T. Gonzalez, M. Bernal, A. Sala, B. Aguiar, Cancellation-based nonquadratic controller design for nonlinear systems via Takagi-Sugeno models, IEEE Transactions on Cybernetics 47 (9) (2017) 2628-2638.

[29] Y. Blanco, W. Perruqueti, P. Borne, Stability and stabilization of nonlinear systems and Takagi-Sugeno fuzzy models, Mathematical Problems in Engineering 7 (3) (2001) 221-240.

[30] K. Tanaka, T. Hori, H. Wang, A multiple Lyapunov function approach to stabilization of fuzzy control systems, IEEE Transactions on Fuzzy Systems 11 (4) (2003) 582-589.

[31] L. Mozelli, R. Palhares, G. Avellar, A systematic approach to improve multiple Lyapunov function stability and stabilization conditions for fuzzy systems, Information Sciences 179 (8) (2009) 1149-1162.

[32] T. Guerra, M. Bernal, A way to escape from the quadratic framework, in: Proceedings of the IEEE International Conference on Fuzzy Systems, Jeju, Korea, 2009, pp. 784-789.

[33] M. Bernal, T. M. Guerra, Generalized non-quadratic stability of continuous-time Takagi-Sugeno models, IEEE Transactions on Fuzzy Systems 18 (4) (2010) 815-822.

[34] A. Sala, C. Ariño, Asymptotically necessary and sufficient conditions for stability and performance in fuzzy control: Applications of Polya's theorem, Fuzzy Sets and Systems 158 (24) (2007) 2671-2686.

[35] G. Chesi, On the gap between positive polynomials and SOS of polynomials, IEEE Transactions on Automatic Control 52 (6) (2007) 10661072 .

[36] K. Tanaka, H. Yoshida, H. Ohtake, H. Wang, A sum-of-squares approach to modeling and control of nonlinear dynamical systems with polynomial fuzzy systems, IEEE Transactions on Fuzzy Systems 7 (4) (2009) 911922 .

[37] A. Papachristodoulou, S. Prajna, Analysis of non-polynomial systems using the sum of squares decomposition, in: Positive polynomials in control, Vol. 312 of Lecture Notes in Control and Information Science, Springer, 2005, pp. 23-43. 
[38] E. D. Andersen, K. D. Andersen, The mosek interior point optimizer for linear programming: An implementation of the homogeneous algorithm, in: H. Frenk, K. Roos, T. Terlaky, S. Zhang (Eds.), High Performance Optimization, Vol. 33 of Applied Optimization, Springer US, 2000, pp. 197-232.

[39] J. Löofberg, YALMIP : A toolbox for modeling and optimization in MATLAB, in: Proceedings of the 2004 IEEE International Symposium on Computer Aided Control Systems Design, Taipei, Taiwan, 2004, pp. 284-289.

[40] H. Lam, Stabilization of nonlinear systems using sampled-data outputfeedback fuzzy controller based on polynomial-fuzzy-model-based control approach, IEEE Transactions on Systems, Man, and Cybernetics, Part B: Cybernetics 42 (1) (2012) 258-267. 